\title{
PSYCHIATRIC DISORDERS AMONG MEDICAL STUDENTS: HOW AWARE ARE WE?
}

\author{
Ranjan S. ${ }^{1}$, Shah S. ${ }^{2}$, Aryal S. ${ }^{2}$, Aich T.K. ${ }^{3}$
}

\begin{abstract}
Studying medicine is a physically and emotionally demanding course. Several studies have documented that medical students are at high risk for developing psychiatric disorders. Also the risk increases because these students who are the future health care providers may misunderstand and stigmatize psychiatric disorders. This can be potentially tragic. This article reviews the data on prevalence of psychiatric disorders among medical students. PubMed and Medlinewere searched to identify latest peer-reviewed English-language studies reporting on depression, anxiety, suicide and other psychiatric disorders among medical students from different parts of the world. Searches were done using the key words medical students, psychiatric disorders, depression, burnout and suicide. References of the retrieved articles were inspected toidentify relevant additional articles. The studies suggest a high prevalence of burnout, depression, suicide and other psychiatric disorders among medical students, higher than in the general population. Medical students are reluctant to seek psychiatric treatment more than the general population. The environment where they study may make them behave in the unprofessional way rather than the mental illness itself. Medical school is a time of significant psychological distress. Large, prospective, multicenter studies are needed to identify personal and training-related features that influence depression, anxiety, and burnout among students and explore relationships between distress and competency.
\end{abstract}

KEY WORDS: Medical students, Psychiatric disorders, Burnout, Suicide and Depression.

1. Associate Professor, Department of Psychiatry, Universal College of Medical Sciences \& Teaching Hospital, Bhairahawa, Nepal

2. Post Graduate Resident, Department of Psychiatry, Universal College of Medical Sciences \& Teaching Hospital, Bhairahawa, Nepal

3. Professor and Head, Department of Psychiatry, Universal College of Medical Sciences \& Teaching Hospital, Bhairahawa, Nepal

\author{
For Correspondence \\ Dr. Sanjeev Ranjan, \\ Associate Professor, \\ Department of Psychiatry, \\ Universal College of Medical Sciences and Teaching Hospital, \\ Bhairahawa, Nepal \\ E-mail:drsanjeevranjan@yahoo.com
}




\section{INTRODUCTION}

Medical students frequently undergo severe stress and fall victim to psychiatric disorders. This may lead to poor performance not only in their academics but also in patient care. Some of them may suffer from serious mental illnesses during their study period and drop their course. Under these circumstances treatment and counselling by psychiatrists is specifically advised. Medical students in general are reluctant to seek advice because of the worries related to confidentiality and stigma associated with mental illness. Research suggests that medical students have higher rates of mental disorder than students pursuing other courses. Many have a substance abuse problem as well. Suicide rates are also high among them. Among the risk factors and sources of stress that lead to mental health problems are: study pressure, high demand of competency, poor study environment and poor relationship with colleagues. ${ }^{1}$ However, genetic vulnerability and other social factors are also important factors in the development of psychiatric disorders. Every individual is vulnerable to physical as well as mental illnesses and so are the people who are pursuing the course in treatment of such illnesses.

Retrieving knowledge about presence of psychiatric conditions is important in itself and if found, would need attention. In addition it might indicate vulnerability that medical school could not be held responsible for. ${ }^{2}$

While burnout or stress-reactions are conceived of as reactions to a pressing environment, psychiatric disorders such as bipolar disorder, depression, anxiety are considered multifactorial involving genetic vulnerability, neurotransmitters and other abnormalities in brain functioning, environmental and social causes.

This is important here because some studies have shown that medical students had higher rates of depression than the general population even before entering medical school. ${ }^{2}$

Whether the demands of medical education or dispositional factors of the individuals choosing this career has made the prevalence of psychiatric disorders in medical students high need to be known.

The knowledge about it will help the universities running medical courses more aware about it and develop strategies to prevent it. It will help the students be aware about the problem and take early treatment.

\section{HOW PREVALENT IS PSYCHIATRIC DISORDERS AMONG MEDICAL STUDENTS?}

Study done at Department of Clinical Neuroscience, Division of Psychiatry St. Göran, Karolinska Institute, Sweden , showed that $25 \%(\mathrm{~N}=81)$ of the medical students in their study had a psychiatric diagnosis. Among them only 6 had sought help. Unadjusted analyses showed that psychiatric morbidity was predicted by high Performance-based self-esteem, disengagement and depression at $1^{\text {st }}$ year. On adjusted analysis, only the later remained significant.

All medical students at seven US schools were surveyed (published in 2011), with standardized instruments to evaluate burnout, depression, stress, mental Quality Of Life (QOL), physical QOL, fatigue, recent suicidal ideation and serious thoughts of dropping out of medical school. Nearly all $(1846 / 2246,82 \%)$ of medical students had at least one form of distress with 1066 (58\%) having $\geq 3$ forms of distress. A doseresponse relationship was found between the number of manifestations of distress and recent suicidal ideation or serious thoughts of dropping out. Those having more forms of distress were more likely to have suicidal ideation than students with no forms of distress.

Burnout is traditionally viewed as a reactive pattern towards negative working conditions, affecting vitality and emotions towards the work. ${ }^{2}$

Another study in USA surveyed medical students $(\mathrm{N}=3080)$ at 5 medical schools in 2006 using validated instruments to assess burnout, depression, and QOL. Students were also asked about the impact of race/ethnicity on their training experience. Nearly half of students reported burnout (47\%) and depressive symptoms (49\%). Mental QOL scores were lower among students than among the age-matched general population (43.1 vs 47.2; $\mathrm{P}<.001$ ). Prevalence of depressive symptoms was similar regardless of minority status, but more nonminority students had burnout $(39 \%$ vs $33 \%$; $\mathrm{P}<.03)$. Minority students were more likely to report that their race/ethnicity had adversely affected their medical school experience $(11 \%$ vs $2 \% ; \mathrm{P}<.001)$ and cited racial discrimination, racial prejudice, feelings of isolation, and different cultural expectations as causes. Minority students reporting such experiences were more likely to have burnout, depressive symptoms, and low mental QOL scores than were minority students without such experiences $($ all $\mathrm{P}<.05){ }^{4}$

Psychiatry and Medical Psychology Disciplines, ABC Regional Medical School at Santo André, Brazil aimed to investigate the characteristics of depressive symptoms among 481 medical students. Medical education and training in Brazil is divided into basic (1 st and 2 nd years), intermediate ( $3 \mathrm{rd}$ and 4th years), and internship (5th and 6th years) periods. The Beck Depression Inventory (BDI) and cluster analyses were used in order to better describe the characteristics of depressive symptoms. The study organized each item from the BDI into the following three clusters: affective, cognitive, and somatic. There were $184(38.2 \%)$ students with depressive symptoms $(\mathrm{BDI}>9)$. The internship period resulted in the 
highest BDI scores in comparison to both the basic $(\mathrm{p}<.001)$ and intermediate $(\mathrm{p}<.001)$ periods. Affective, cognitive, and somatic clusters were significantly higher in the internship period. An exploratory analysis of possible risk factors showed that females $(\mathrm{p}=.020)$ not having a parent who practiced medicine $(\mathrm{p}=.016)$, and the internship period $(\mathrm{p}=$ .001 ) were factors for the development of depressive symptoms.

To assess the prevalence of suicidal ideation among university students, a study was done at the department of psychiatry, in Colombia; which found that the prevalence of the current suicidal ideation was $3.6 \%$, and $18 \%$ in the last year. Both suicidal ideation and history of suicide attempts were higher among female students. Rape history, being alone more than eight hours a day, and female gender were the factors more strongly related to suicidal ideation. A relationship between suicidal ideation and awareness of the suicide of others was not found in this sample. ${ }^{6}$

Between 2007 and 2010 all 1112 students entering the Core Science component (Year 1) and all 542 students entering the Clinical component (Year 4) of the Cambridge (UK) medical course were followed-up annually. Mean HADS-D scores, the proportions whose scores indicated depression at different time-points and for students maintaining participation, the number of occasions on which their HADS-D scores indicated depression were analysed separately for men and women.725 Core Science and 364 clinical students participated. Mean HADS-D scores ranged between 3.34 and 3.49 among all core science students and between 2.16 and 2.91 among all clinical students. There was no difference between men and women in median HADS-D scores. Prevalence of depression ranged between $5.7 \%$ and $10.6 \%$ among all core science students and between $2.7 \%$ and $8.2 \%$ among all clinical students. Over time core science students displayed no increase in mean HADS-D score. Among clinical students only men displayed a small increase (time coefficient 0.33 (95\% CI 0.11 to 0.55$)$ ). Prevalence did not increase over time. 220 core science and 150 clinical students participated throughout the study. Of these, $18.2 \%$ and $10.6 \%$ respectively, recorded HADS-D scores indicating depression on at least one occasion.

A study was done by MRC/ESRC Social and Applied Psycology Unit, University of Sheffield. Among 170 junior house officers who were followed up from their fourth year in medical school mean levels of stress were higher than in other reported occupational groups, and the estimated prevalence of emotional disturbance was $50 \%$, with $28 \%$ of the subjects showing evidence of depression. Nearly a fifth of the subjects reported occasional or frequent bouts of heavy drinking, a quarter took drugs for physical illness, and a few took drugs for recreation. Those who were emotionally distressed at the initial study and the follow up were more empathetic and more self critical than those who had low levels of stress on both occasions. ${ }^{8}$

A cross sectional study using self administered questionnaire was conducted among a sample of undergraduate students chosen from all the years of MBBS who were enrolled at Govt. Medical College, Surat at the time of study period. In this study $3.12 \%$ of the students reported having no stressor experience, $55.6 \%$ reported mild to moderate stressor experience and $41.2 \%$ with severe stressor experience. Major factors responsible for stress identified in this study were increased load towards exam, vast syllabus, not getting expected marks, less time for repeated learning and procrastination. ${ }^{9}$

\section{FEAR OF STIGMATIZATION AMONG MEDICAL STUDENTS}

To characterize the perceptions of depressed and nondepressed medical students regarding stigma associated with depression, cross-sectional web-based survey was conducted in September-November 2009 among all students enrolled at the University of Michigan Medical School $(\mathrm{N}=$ 769). Prevalence of moderate to severe depression was $14.3 \%$ (95\% confidence interval [CI], 11.3\%-17.3\%). Women were more likely than men to have moderate to severe depression (18.0\% vs $9.0 \%$; $95 \%$ CI for difference, $-14.8 \%$ to $-3.1 \% ; P=$ $.001)$. Third and fourth year students were more likely than first and second year students to report suicidal ideation $(7.9 \%$ vs $1.4 \% ; 95 \%$ CI for difference, $2.7 \%-10.3 \% ; P=.001$ ). Students with moderate to severe depression, compared with no to minimal depression, more frequently agreed that "if I were depressed, fellow medical students would respect my opinions less" (56.0\% vs $23.7 \%$; $95 \%$ CI for difference, $17.3 \%-47.3 \% ; P<.001)$, and that faculty members would view them as being unable to handle their responsibilities (83.1\% vs 55.1\%; 95\% CI for difference, 16.1\%-39.8\%; $P<$ $.001)$. Men agreed more commonly than women that depressed students could endanger patients (36.3\% vs $20.1 \%$; $95 \%$ CI for difference, $6.1 \%-26.3 \% ; P=.002)$. First and second year students more frequently agreed than third and fourth year students that seeking help for depression would make them feel less intelligent $(34.1 \%$ vs $22.9 \%$; $95 \%$ CI for difference, $2.3 \%-20.1 \% ; P<.01){ }^{10}$

This suggested that depressed medical students more frequently endorsed several depression stigma attitudes than nondepressed students. Stigma perceptions also differed by sex and class year.

Stigma associated with depression and the use of mental health care services may represent a barrier to seeking treatment. One study identified stigmas an explicit barrier to the use of mental health services by $30 \%$ of first and second year medical students experiencing depression. In addition, $37 \%$ identified 
lack of confidentiality and $24 \%$ cited fear of documentation in their academic record as barriers to treatment. Students may worry that revealing their depression will make them less competitive for residency training positionsor compromise their education. ${ }^{11}$

If medical students are critical of each other about depression, It is doubtful that they can help patients with depression. We don't want the medical education experience to make them less tolerant of mental illness. Stigma seems to be lessening among the general public, but the medical professional is lagging behind.

Medical students' attitudes towards suicide and suicidal patients were studied at Department of Psychiatry, SouthEastern Health Region, Stockholm, Sweden. The aim was also to determine whether attitudes differ between students in the beginning and end of studies. A questionnaire including own attitudes on death and suicide and psychosocial circumstances was filled in by $63 \%$ of first and final year students (306 of 485). The calculation included a factor analysis on items describing the attitudes to suicidal patients. Attitudes towards patients became influenced by the knowledge of mental disorders and by biological aspects of behaviour during the education. Final year students more often consider suicide to be an expression of psychiatric disease and thought that people trying to commit suicide were not responsible for their own actions. Thirty four percent and $44 \%$ (ns) in the first and last years, respectively, reported suicidal ideas some time in their lives. Students with such a history of suicide thoughts were less optimistic about the possibility to help. Ongoing depressive/anxious symptoms were prevalent in 36/305 (12\%) of students, but did not seem to affect their attitudes to patients. Female students had sought psychological/psychiatric help more often than males $(26 \%$ and $10 \%, \mathrm{P}<0.01){ }^{1}$

\section{EFFECT ON BEHAVIOUR}

Burnout and psychiatric disorders in medical students can lead to unprofessional behaviour. Dyrbye and colleagues surveyed more than 2500 medical students. His work published in the Journal of the American Medical Association (JAMA) in September, 2010 showed important differences between professional and personal distress and their effects on behaviours. Burnout or emotional exhaustion and detachment led students to behave unprofessionally, such as cheating in tests, and feeling less altruistic about their role as physicians. However, personal distress, caused by depression and poor mental quality of life, was less likely to lead to unprofessional behaviours. The association between cheating/dishonest clinical behaviors and students' views regarding physicians' responsibility to society is more specific to burnout (ie, professional distress) rather than simply distress, according to the authors. "The fact that students frequently engage in dishonest behaviours despite knowing they are inappropriate may imply that some elements of the learning climate foster dishonesty. ${ }^{12}$

\section{INTERVENTIONS}

Although depressive symptoms and suicidal ideation are common in medical students, there are few programs to address this serious problem.

To reduce the rates of depression and suicidal ideation among medical students, the University of Hawaii John A. Burns School of Medicine implemented the following interventions: increased individual counseling for students, faculty education, and a specialized curriculum including lectures and a student handbook. Although counseling had always been available, a new emphasis was placed on facilitating an anonymous process and providing several options, including volunteer psychiatrists not involved in student education. In 2002 and 2003, the authors measured depressive symptoms and suicidal ideation in third-year medical students using, respectively, the Center for Epidemiologic Studies Depression Scale and a question about suicidal ideation from the Primary Care Evaluation of Mental Disorders Patient Health Questionnaire. Before the intervention, 26 medical students (59.1\%) had reported depressive symptoms, and 13 (30.2\%) reported suicidal ideation. After the intervention, 14 medical students $(24.1 \%)$ reported depressive symptoms $\left(\chi^{2}=12.84\right.$, $\mathrm{df}=2, \mathrm{P}<.01)$, and $1(3 \%)$ reported suicidal ideation $\left(\chi^{2}=\right.$ $13.05, \mathrm{df}=1, \mathrm{P}<.001)$

This suggests that programs that provide specific mental health support for medical students may significantly decrease the reported rates of depressive symptoms and suicidal ideation.

\section{CONCLUSION}

Majority of medical students suffer from some form of psychiatric disorders. Academic, environmental and emotional factors together are responsible for this stress. The concerned health system may suffer because of this problem as there will be absenteeism, poor academic performance, unprofessional behaviours and drop out from course. It is important to either prevent or manage this problem. Fear of stigma is more among medical students than general patients which leads to undertreatment of these disorders among the medical students. Proper guidance and counseling by faculties may help to improve the present scenario. Health system policy makers should be aware of the fact that nobody is immune to mental illness in this present era of competition and chaos. 


\section{RECOMMENDATIONS}

The authors recommend that there should be mandatory monthly counselling for all medical students in order to detect and treat psychiatric disorders at the very beginning stage.

The selection committees for medical schools should select candidates who are not only academic achievers but at the same time should have stable personalities.

Data and research are urgently needed so that medical schools could better prepare and protect their students. Somehow we have to change the environment in which we are teaching the future doctors.

Medical students should seek psychiatric help for any mental health problem and remain aware of their vulnerability especially if there is family history, modify their life style, devise a stress free study schedule and convince themselves that it is not a stigma to seek help for mental problem.

The concerned medical colleges and universities should avoid stigmatizing those students who become mentally ill.

\section{REFERENCES}

1. Amin A. Muhammad G. Mental illness among doctors: How serious can this be? May 2009

2. Dahlin ME, Runeson B. Burnout and psychiatric morbidity among medical students entering clinical training: a three year prospective questionnaire and interview-based study. BMC Medical Education2007,7:6 doi:10.1186/1472-6920-7-6

3. Dyrbye LN, Harper W, Durning SJ, Moutier C, Thomas MR, Massie FS Jr, Eacker A, Power DV, Szydlo DW, Sloan JA, Shanafelt TD "Patterns of distress in US medical students". Med Teach. 2011;33(10):834-9. doi: 10.3109/0142159X.2010.531158.

4. Dyrbye LN, Thomas MR, Eacker A, Harper W, Massie FS Jr, Power DV, Huschka M, Novotny PJ, Sloan JA, Shanafelt TD.
"Race,Race, ethnicity, and medical student well-being in the United States".Arch Intern Med. 2007 Oct 22;167(19):2103-9.

5. Baldassin S, Alves TC, de Andrade AG,Nogueira Martins LA. "The characteristics of depressive symptoms in medical students during medical education and training: a crosssectional study".BMC Med Educ. 2008 Dec 11;8:60. doi: 10.1186/1472-6920-8-60

6. Rev SaludPublica (Bogota). "Prevalence and factors associated with suicidal thinking among university students".2003 MayAug;5(2):123-43

7. Quince TA, Wood DF, Parker RA, Benson J. "Prevalence and persistence of depression among undergraduate medical students: a longitudinal study at one UK medical school". BMJ Open. 2012 Aug 13;2(4). pii: e001519. doi: 10.1136/bmjopen-2012-001519.

8. Firth-Cozens J. "Emotional distress in junior house officers". Br MedJ(Clin Res Ed).1987 Aug 29;295(6597):533-6.

9. Solanky P, Desai B, Kavishwar A, Kantharia SL. Study of psychological stress among undergraduate medical students of government medical college, Surat. Int JMed Sci Public Health. 2012; 1(2): 38-42doi: 10.5455/ijmsph.2012.1.38-42.

10. Thomas L. Schwenk, MD; Lindsay Davis, BS; Leslie A. Wimsatt, $P h D$ "Depression, Stigma, and Suicidal Ideation in Medical Students". JAMA.2010;304(11):1181-1190.

11. Givens JL, Tjia J. Depressed medical students' use of mental health services and barriers to use. AcadMed. 2002;77(9):918-921.

12. Devi S. "Doctors in distress". The Lancet, Volume 377, Issue 9764, Published by Elsevier Pages 454 - 455, 5 February 2011

13. Wallin U, Runeson B. "Attitudes towards suicide and suicidal patients among medical students". Eur Psychiatry. 2003 Nov;18 (7):329-33.

14. Thompson D, Goebert D, Takeshita J. "A program for reducing depressive symptoms and suicidal ideation in medical students".Acad Med. 2010 Oct;85(10):1635-9. doi: 10.1097/ACM.0b013e3181f0b49c. 